licht das Dilemma der öffentlich-rechtlichen Mediaforschung: Eine Typologie, die Redaktionen, Mediaplaner, Werbekunden und Medienpolitik gleichermaßen bedient, muss erst noch erfunden werden.

Michael Meyen

\section{Henning Never}

\section{Meinungsfreiheit, Wettbewerb und Markt- versagen im Rundfunk}

Eine ökonomische Kritik der verfassungsrechtlich geforderten positiven Rundfunkordnung

Baden-Baden: Nomos, 2002. - $330 \mathrm{~S}$.

(Schriften zur Medienwirtschaft und zum Medienmanagement; 1)

ISBN 3-7890-7865-4

In der vorliegenden Dissertation untersucht Henning Never die normative Grundlage für eine positive Rundfunkordnung und mögliche Wege ihrer Umsetzung. Sie ist als erster Band in einer neuen Reihe „Schriften zur Medienwirtschaft und zum Medienmanagement" erschienen, welche sich nicht - wie im Untertitel angegeben - mit der ,positiven Rundfunkforschung“, sondern wohl eher mit der „positiven Rundfunkordnung" beschäftigen will.

Die Arbeit selbst ist aufgeteilt in elf in sich abgeschlossene Kapitel. Zunächst beschreibt der Autor die Entwicklung der deutschen Rundfunklandschaft mittels rundfunkspezifischer Kennzahlen. Dabei vergleicht er quantitative Größen einzelner Programmgenres im öffentlich-rechtlichen sowie im werbefinanzierten Rundfunk. Anhand dieses Schemas analysiert er qualitative Unterschiede, die im Zuge der Konvergenz privater und öffentlichrechtlicher Programmanbieter mehr und mehr verwischt worden seien und deren Angleichung in naher Zukunft zum Abschluss käme.

In Kapitel 3 interpretiert der Verfasser dezidiert die Entwicklung des Artikel 5 Abs. 1 Satz 2 GG als objektivrechtlich ausgestaltetes Instrumentarium, das sich von einem Schutzrecht in der Übertragungsfreiheit über die verfassungsrechtlich zugesprochene vollständige Sicherung der Gewährleistungsfreiheit des gesamten Meinungsbildungsprozesses hin zu einer Ordnung entwickelt habe, die die „freie (Meinungs-) Konkurrenz als letztlich selbstzerstörerisch“" ablehne. Die so entstandene po- sitive Rundfunkordnung sei-als Resultat einer im Wesentlichen ökonomisch-technischen und weniger einer publizistisch-kommunikationswissenschaftlichen Sondersituation - einfachgesetzlich normiert von staatlicher Seite ausgestaltet worden.

Ausgehend vom evolutorischen Prozess der Wissensgenerierung und dessen Grenzen stellt Never fest, dass die bestehende Rundfunkordnung prinzipiell unvereinbar sei mit dem Prozess freier Meinungsbildung. Dieser zeichne sich als ergebnisoffenes Entdeckungsverfahren gerade durch Meinungsungleichgewichte aus. Es könne keine institutionelle (Ab-)Sicherung neuer Meinungen geben.

In der folgenden, in der Gewichtung der einzelnen Kapitel relativ knapp gehaltenen ökonomischen Analyse und Bewertung stellt der Autor zunächst mögliche Marktversagenstatbestände gegenüber und durchleuchtet diese, bevor er den Einfluss der Finanzierung auf Vielfalt und Qualität der angebotenen Programme interpretiert. Trotz der nicht hinreichenden Erläuterung, dass bereits bei der Normierung der beiden Begriffe Schwierigkeiten und differierende Ansichten bestehen, resümiert Never, dass sich formal höher Gebildete ihren (öffentlich-rechtlichen) Rundfunkkonsum durch Ärmere oder weniger gebildete Rezipienten finanzieren ließen, welche vorwiegend das Angebot der werbefinanzierten Anbieter nutzen würden.

Einen guten Überblick über die Gesetzeslage zum Zeitpunkt der Fertigstellung der Dissertation bieten die Kapitel 7 bis 9, in denen der Verfasser die rechtlichen Grundlagen auf den verschiedenen Entscheidungsebenen (EU, Bund, Länder) sowie entstandene Abgrenzungsprobleme der verschiedenen Übertragungsdienste darstellt. Zwischenzeitlich sind einige Texte allerdings - teilweise in Sinne des Autors - überarbeitet worden (z. B. zur Rundfunkkonzentrationskontrolle, andere Änderungen stehen derzeit zur Diskussion). Never allerdings resümiert, dass eine spezifische Rundfunkkontrolle gänzlich überflüssig sei, da eine Funktionsstörung nicht abschließend eingeordnet werden könne, frei nach dem Motto: Wenn etwas nicht hundertprozentig messbar ist (hier: inhaltliche Vielfalt), dann soll es (beispielsweise über gesetzlich festgelegte Rahmenbedingungen, wie regionale Informationsanteile) auch nicht näherungsweise bestimmt, sondern deren Regelung gänzlich abgeschafft werden. 
In seinem Fazit unterstreicht der Autor einige Vorschläge für eine ordnungspolitische Reform der dualen Rundfunkordnung. So kann seiner Ansicht nach das Angebot öffentlichrechtlicher Rundfunkanbieter beschränkt werden auf rein nicht-marktfähige Güter. Auszustrahlende Programme, so Never, ließen sich dann definieren anhand von Einschaltobergrenzen. Sobald ein Programm die Obergrenze überschreite, dürfte es im öffentlich-rechtlichen Rundfunk nicht mehr gesendet werden. Wer diese Grenze setzen soll, lässt der Verfasser offen, ebenso wie die Erörterung des im Vorschlag enthaltenen Widerspruchs, dass Rundfunkprogramme nach seiner Auffassung uneingeschränkt marktfähig sind, er aber gleichzeitig Programme mit einer geringen Einschaltquote als meritorische Güter betrachtet. Auch die vorgeschlagene Subventionierung unabhängiger Produzenten für nicht-marktfähige Rundfunkangebote bei gleichzeitiger Abschaffung des öffentlich-rechtlichen Rundfunks in der jetzigen Form kann kaum überzeugen. Weder wird erörtert, wer über die Subventionswürdigkeit und deren Höhe entscheiden soll, noch wird der damit verbundene Staatseingriff in die Autonomie privater Veranstalter problematisiert. Insgesamt erscheint das Fazit bei einer weitgehend stringenten Argumentationsstrategie als unausgewogen und nicht ausreichend begründet. Denn die entscheidende Frage, wie auf Basis der begründeten Feststellungen des Verfassers eine Veränderung aus institutioneller Sicht aussehen müsste, wird nicht hinreichend ausgearbeitet.

Der Autor argumentiert zu Recht, dass dem Prozess schöpferischer Zerstörung durch die bestehende Rundfunkordnung infolge der zum Teil dogmatischen Rechtsprechung des Bundesverfassungsgerichts eine enge Grenze gesetzt werde. Dennoch - und gerade das zeigt Nevers Analyse - bleiben ausreichend Entfaltungsspielräume für private wie öffentlichrechtliche Anbieter. Gerade weil im dualen Rundfunksystem eine große Vielfalt herrscht, kommt es nicht zu nivellierenden Tendenzen, sondern zu einer ergebnisoffenen Dynamik des gesellschaftlichen Meinungsbildungsprozesses. Die funktionale Ausgestaltung der verfassungsmäßigen Rundfunkordnung beruht auf einem gesellschaftlichen Konsens, unter dem gesellschaftlich als wichtig empfundene Werte und Tugenden sowie gesellschaftliche Ziele, die es $\mathrm{zu}$ erreichen gilt, subsummiert werden.
Wenn also eine positive Rundfunkordnung auf Basis eines normativen Konzeptes entwickelt werden soll, dann muss der gesellschaftliche Konsens der Ausgangspunkt und Fokus der Analyse sein. Diese ist auch aus ökonomischer Sicht zu rechtfertigen: Wenn ein Individuum einen höheren Nutzen in einem gesellschaftlich bereitgestellten Angebot sieht, dann ist es eine „Anmaßung von Wissen“, ihm dieses Angebot abzusprechen. Auch ein Rückgriff auf die Konstrukte „meritorische Güter" und „intransitive Präferenzen" ist dann nicht mehr notwendig. Die duale Rundfunkordnung ist letztlich eine auf Individualentscheidungen beruhende gesellschaftliche Selbstbindung. Sowohl Ausgestaltung als auch Umfang beruhen auf dieser Entscheidung, auf deren Basis freilich noch ausreichend viele Erkenntnisse - ganz im Sinne Nevers - akkumuliert werden können.

Eine Schwäche ist die zum Teil statische Sichtweise des Verfassers, die den dynamischen Charakter gesellschaftlicher Evolution weitgehend vernachlässigt und die wettbewerbliche Dynamik innerhalb der vorgegebenen Rundfunkordnung zu wenig beachtet. Obwohl der Verfasser selbst dem Bundesverfassungsgericht bereits in den einleitenden Worten eine dynamische Sichtweise der verfassungsrechtlich gebotenen Auslegung der Rundfunkfreiheit abverlangt, prognostiziert er dem öffentlichrechtlichen Rundfunk sinkende Zuschauerzahlen (die seiner Meinung nach den einzigen Indikator für die Akzeptanz öffentlich-rechtlichen Programmangebots bilden) als exogenstatische Entwicklung, ohne ihm die Möglichkeit einzuräumen, diesem Trend entgegenzuwirken.

Never stellt bereits frühzeitig fest, dass der „Wettstreit der Hypothesen“ niemals zu einem Ende geführt werden kann, da Hypothesen lediglich Vermutungen darstellen. Wenn aber das Bundesverfassungsgericht ein - so Never - rein auf ihren Hypothesen beruhendes Rundfunkkonstrukt entwickelt hat, so stellt sich die Frage, ob sich Never mit seinen Handlungsvorschlägen nicht diese Erkenntnis zu eigen macht. Der Autor analysiert sauber, aber die Schlussfolgerung ist auf ein Ergebnis ausgerichtet, das nicht auf den eigenen Erkenntnissen beruht und zum Teil den eigenen Zwischenergebnissen widerspricht.

Stefan Ollig 